\title{
Regulation of bolting and identification of the a-tubulin gene family in Brassica rapa L. ssp pekinensis
}

\author{
Y.W. Zhang ${ }^{1,2}$, D. Jin ${ }^{1}$, C. Xu' ${ }^{1}$, L. Zhang ${ }^{1}$, M.H. Guo ${ }^{1}$ and Z.Y. Fang ${ }^{3}$ \\ ${ }^{1}$ College of Horticulture, Northeast Agricultural University, Harbin, China \\ ${ }^{2}$ Key Laboratory of Biology and Genetic Improvement of Horticultural Crops \\ (Northeast Region), Ministry of Agriculture, Harbin, China \\ ${ }^{3}$ Institute of Vegetables and Flowers, Chinese Academy of Agricultural Sciences, \\ Beijing, China
}

Corresponding author: Y.W. Zhang

E-mail: zhangyaowei@neau.edu.cn

Genet. Mol. Res. 15 (1): gmr.15017507

Received August 24, 2015

Accepted November 6, 2015

Published January 29, 2016

DOI http://dx.doi.org/10.4238/gmr.15017507

ABSTRACT. Microtubules are important components of eukaryotic cells, and they play vital roles in cell morphogenesis, carrying of signaling molecules, transport of materials, and establishing the cell polarity. During bolting of biennial plants, cell division and elongation are involved, and cell elongation inevitably involves the microtubules arrangement and expression of related genes. So we deduce that it is of great significance to figure out the mechanism of bolting and flowering in which TUA genes are involved. In the present study, bioinformatic methods were used to predict and identify the a-tubulin gene family (BrTUAs) in Brassica rapa L. ssp pekinensis (Chinese cabbage) through the alignment of AtTUA gene sequence from Arabidopsis thaliana with the $B$. rapa genome database (http://brassicadb.org/brad/) using the basic local alignment search tool. The change in the structure and functions of BrTUAs during the process of evolution, cis-acting elements in the promoter sequences of BrTUAs, and the expression of the identified genes was also analyzed. Twelve members 
of the a-tubulin gene family were identified from Chinese cabbage. The gene length, intron, exon, and promoter regions were determined to have changed significantly during the genome evolution. Only five of the 12 members were encoded completely and were observed to differ in their spatial and temporal expression. The five BrTUA promoter sequences contained different numbers of cis-elements responsive to light and lowtemperature response, cis-elements responsive among which hormonal responses were significantly different. We also report that the BrTUAs were involved in the regulation of the bolting in Chinese cabbage, and propose that this process could be controlled by regulating the expression of BrTUAs.

Key words: Brassica rapa; a-tubulin gene; Identification; Expression

\section{INTRODUCTION}

Microtubules, which are important components of eukaryotic cells, play vital roles in cell morphogenesis, carrying of signaling molecules, transport of materials, the establishment of polarity of cells and in other aspects (Chan et al., 2011). It is necessary for plant cells to have different morphology to adapt to the environment and functional needs during the process of growth and development. These processes are closely associated with the microtubules. Kopczak et al. (1992) reported that there were at least six TUA gene subtypes and nine TUB gene subtypes in Arabidopsis thaliana. They also separated some TUA and TUB gene subtypes from different plants: at least eight $\alpha$-microtubules and seven $\beta$-microtubules subtypes in grapes (Parrotta et al., 2010), at least eight $\alpha$-microtubules and twenty $\beta$-microtubules subtypes in poplar (Oakley et al., 2007), and nine a-microtubules subtypes in cotton (Dixon et al., 1994). The TUA genes of $A$. thaliana are highly conserved genetically and are very similar in structure, but they are considerably different in expression. The TUA genes 2, 3, 4, and 5 of $A$. thaliana express in roots, stems, and leaves, and TUA genes 1 and 6 express only in flowers (Kopczak et al., 1992).

During bolting of biennial plants, cell division and elongation are involved. Cell elongation inevitably involves the microtubules arrangement and expression of related genes. It is, therefore, of great significance to understand the mechanism of bolting and flowering in which the TUA genes might be involved. Chinese cabbage is a typical biennial plant that has undergone triploidization and double-ploidization during the evolutionary process as a new polyploidy (Cheng et al., 2013). According to the results of Brassica rapa genome sequencing, there are massive repeated gene fragment in B. rapa genome, which might contain multiple TUA gene subtypes. In the present study, $\alpha$-tubulin gene family was identified and the effects of $B$. rapa polyploidization on the structure, function, and expression patterns of TUA genes were determined. The results obtained might help to understand the regulation of bolting and flowering of Chinese cabbage by the TUA genes.

\section{MATERIAL AND METHODS}

\section{Plant material}

An advanced generation inbred line of Chinese cabbage (A161) having uniform botanical characteristics, bolting, and early flowering, bred by the Chinese Cabbage Research Group of the Northeast Agricultural University was used in the present study. 


\section{Structure, evolution, and identification of BrTUA genes}

Amino acid sequences of six TUA genes of $A$. thaliana were used as queries in the BLAST analysis against the $B$. rapa genome database (http://brassicadb.org/brad/). BLASTp was chosen as operation procedure to run Blast program online and B. rapa (protein) as database. For different parameters, the default values were selected. The sequences of redundant hits and those withan $\mathrm{E}$ value less than -10 and similarity less than $60 \%$ were removed; the remaining sequences were considered the identified sequences. The online analysis software Gene Structure Display Server2.0 (GSDS2.0) (http://gsds.cbi.pku.edu.cn) was used to determine the exon and intron regions. The Mega 5.1 software was used for sequence alignment and construction of a molecular phylogenetic tree using the mRNA and amino acid sequence of BrTUAs and TUAs of $A$. thaliana and other species. For functional analysis of the protein, tools (InterPro) available at EMBL-EBI website (http://www.ebi.ac.uk/interpro/) were used.

\section{Analysis of cis-elements in the promoter sequences of the BrTUA genes}

To investigate the cis-elements in the promoter sequences of BrTUA genes, genomic DNA sequences located $1.5 \mathrm{~kb}$ upstream of the initiation codon (ATG) for each gene were obtained from the Brassica database BRAD. The PlantCARE database (http://bioinformatics.psb.ugent.be/ webtools/plantcare/html/) was used to identify the cis-elements in the promoter regions.

\section{Analysis of expression of BrTUA genes by reverse transcription-polymerase chain reaction (RT-PCR)}

Test seeds were sown in $8 \times 8 \mathrm{~cm}$ seedling pots. The seedlings were subjected to vernalization in an illuminated incubator after the cotyledons were laid fully-open at $8 / 3^{\circ} \mathrm{C}$ (day/night) with $16 / 8$-h (light/dark) period for 25 days. Thereafter, the vernalized plants were transplanted on May 20, 2014 to an open field to receive optimum temperature and long-day for normal growth. Fifteen days after flowering, the new young leaves smaller than $1 \mathrm{~cm}$, tender scape smaller than $2 \mathrm{~cm}$, flower buds one day before flowering, and new fibrous roots and pods seven days after pollination were collected and flash-frozen in liquid nitrogen for the analysis of the difference in the spatial expression of TUA. We collected the shortening stems before the low-temperature vernalization, after vernalization, and during the color-change period (Figure 1) (a change in leaf-color from bright-green to gray-green was observed after the flower bud differentiation and before bolting in the Chinese cabbage) (Han et al., 2011) and scape with flower stalk up to $2 \mathrm{~cm}$ and then flash-frozen in liquid nitrogen for subsequent expression analysis of TUA during the bolting process.

For the expression analysis of TUAs using RT-PCR, 12 primers were designed (Table 1). The forward and reverse primers were designed, respectively, against the coding DNA sequence (CDS) and the 5'-untranslated region sequences that were determined to be highly similar among the BrTUAs. RNA was extracted using the EasyPure ${ }^{\circledR}$ Plant RNA Kit (TransGen Biotech Co., Ltd., Beijing, China) and was stored at $-80^{\circ} \mathrm{C}$, for subsequent use. The synthesis of the first strand cDNA was performed by reverse transcription using TransScript ${ }^{\circledR}$ First-Strand cDNA Synthesis SuperMixKit (TransGen Biotech Co., Ltd.) by incubating the reaction mixture at $42^{\circ} \mathrm{C}$ for 30 min followed by heating at $85^{\circ} \mathrm{C}$ for $5 \mathrm{~min}$ to inactivate the RNA. The RT-PCR product was analyzed by agarose gel electrophoresis. GADPH was used as the reference gene. 


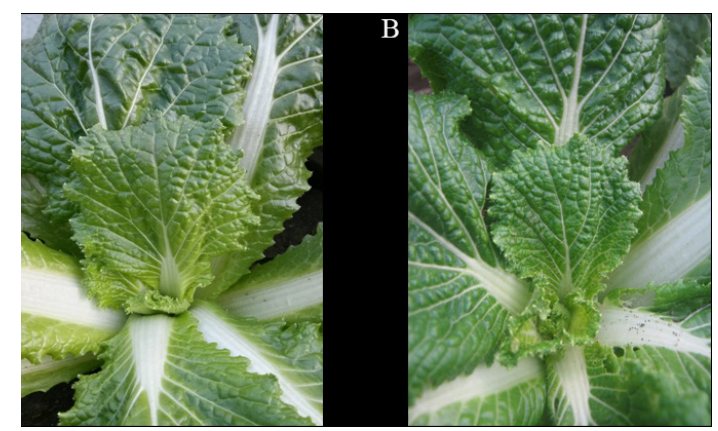

Figure 1. Change in leaf color before bolting in Chinese cabbage. Leaf color before (A) and after (B) bolting.

Table 1. Specific primer of BrTUAs used for RT-PCR.

\begin{tabular}{|c|c|c|}
\hline Gene & Forward primer & Reverse primer \\
\hline Bra039648 & 5'- GAGTGTTGCTGAGGTGTTC-3' & 5'-AAAGAAATAAATCGGATAAGTAGTTTG-3' \\
\hline Bra022914 & 5'-GGACAACCTTGATCGGATACTC-3' & 5'-CCACCTTCAGCACCAACC-3' \\
\hline Bra020572 & 5'-TCCTCCTACGCACCAGTG-3' & 5'-TCCTCGCCTTCGTCATCC-3' \\
\hline Bra018825 & 5'-ATCAACTACCAGCCACCAAC-3' & 5'-TCAGACAGCAAGAGACAGATAG-3' \\
\hline Bra020061 & 5'-GTATCAACTACCAACCTCCAAC-3' & 5'-CGAACCAAGATAATAACAGAACG-3' \\
\hline Bra020062 & 5'-CAAGACTAAGAGGACCGTTCAG-3' & 5'-GTAATCCACAAGAATCATAAGTAGAAAC-3' \\
\hline Bra018176 & 5'-ATGCGTCTGTTTCGGTTTTG-3' & 5'-AAGAATCAGTAAGAAGAAGAGATAAGG-3' \\
\hline Bra008412 & 5'-GCTCCTCCACCGTTCAAG-3' & 5'-GGACCATAACCGCAACAG-3' \\
\hline Bra006517 & 5'-TTAATGCTGCTGTTGGACTATC-3' & 5'-AAGACGGTGGAGAATGTGC-3' \\
\hline Bra002261 & 5'-TGGTGAAGGAATGGAGGAAG-3' & 5'-GTAAGAAGATAATGGAAGGTTTGG-3' \\
\hline Bra002260 & 5'-AAGATGTTAATGCTGCTGTTGG-3' & 5'-TACCTCCTCATAGTCCTTCTCC-3' \\
\hline Bra014232 & 5'-CAAGTGTTGCTGAGGTGTTC-3' & 5'-CACAAATAGAGATGGATAGTAATAACC-3' \\
\hline
\end{tabular}

\section{Gibberellic acid $3\left(\mathrm{GA}_{3}\right)$, methyl jasmonate $(\mathrm{MJ})$, and taxol treatments}

To investigate the way of regulation bolting and flowering in Chinese cabbage, the effect of tubulin specific drug, taxol, as well as $\mathrm{GA}_{3}$ and $\mathrm{MJ}$ on the expression of BrTUA was determined by spraying their solutions on the vegetative growing point of Chinese cabbage near the time of color change. The $\mathrm{GA}_{3}$ and MJ solutions were prepared in ethanol and diluted to 200 (Dai et al., 2010) and $10 \mathrm{mg} / \mathrm{L}$ (Lin et al., 2011) with distilled water. Taxol was dissolved in dimethyl sulfoxide and diluted to $0.3 \mathrm{mg} / \mathrm{L}$ with distilled water (Thitamadee et al., 2002). Distilled water as used as a control. The gene expression was analyzed using RT-PCR after $24 \mathrm{~h}$. For each treatment, the height of floral axis was also determined at the time when it was $5 \mathrm{~cm}$ for the control group.

\section{RESULTS}

\section{Identification and isolation of the TUA genes in $B$. rapa}

To identify the members of the TUA gene family in B. rapa L. ssp pekinensis, sequences of all the TUA genes and their homologs in A. thaliana were retrieved from GenBank. The orthologs of 
A. thaliana genes in $B$. rapa could be obtained from BRAD, a data base containing $B$. rapa genes. Each TUA gene loci in $A$. thaliana was used to search all the TUA gene sequences of $B$. rapa present in BRAD. Each predicted $B$. rapa TUA gene sequence was confirmed using FGENESH (http://www.softberry.com/berry.phtml?topic=fgenesh). The overall analysis revealed that the BrTUA gene family comprised of 12 members (Table 2). The different BrTUA genes varied in their length (from 1007 bp for Bra022914 to 3690 bp for Bra020062) and in the length of the ORFs (from $258 \mathrm{bp}$ for Bra008412 to 1380 bp for Bra006517). The ORFs encoded polypeptides containing 85 to 450 aa with their predicted molecular mass ranging from 21.53 to $116.52 \mathrm{kDa}$.

The BrTUAs CDS and the corresponding genome sequence were used to determine the number and location of introns and exons using the GSDS webset. During the lengthy evolutionary process of BrTUA gene family, most of sequence was determined to have retained four introns whereas the Bra018176, Bra008412, and Bra022914 genes were observed to have lost the exons and introns (Figure2). The length of exons and introns in the 12BrTUA genes varied widely with the introns ranging from 65 to $2100 \mathrm{bp}$ and the exons from 48 to $654 \mathrm{bp}$. This demonstrated that the exon junction components were more varied among the different copies during the evolution of BrTUA. Moreover, we found that intron phases were more abundant, which contained 0, 1, and 2 phases. Of these three phases, the 0 phase was most abundant with just Bra020572 containing a single 0 phase; a 128-bp regulatory sequence was inserted in front of the first codon.

The chromosomal localization and transcription directions of the 12 BrTUA genes (copies) were determined using the genome browse tool in BRAD. As shown in Table 2, the BrTUA genes were distributed on 5 chromosomes; Bra039648 was, however, not anchored on chromosomes, but located between the 212,440-214,331 bp of the scaffold 000172. Four BrTUA genes were located on chromosome 2, and two BrTUA gene copies, each, were present on chromosomes 3, 6, and 10. One BrTUA gene was found on chromosome 8. The BrTUA genes formed 2 clusters with Bra020061 in one cluster and Bra020061, Bra002260, and Bra002261 in the other, indicating that tandem duplication occurred during the evolution of BrTUA genes.

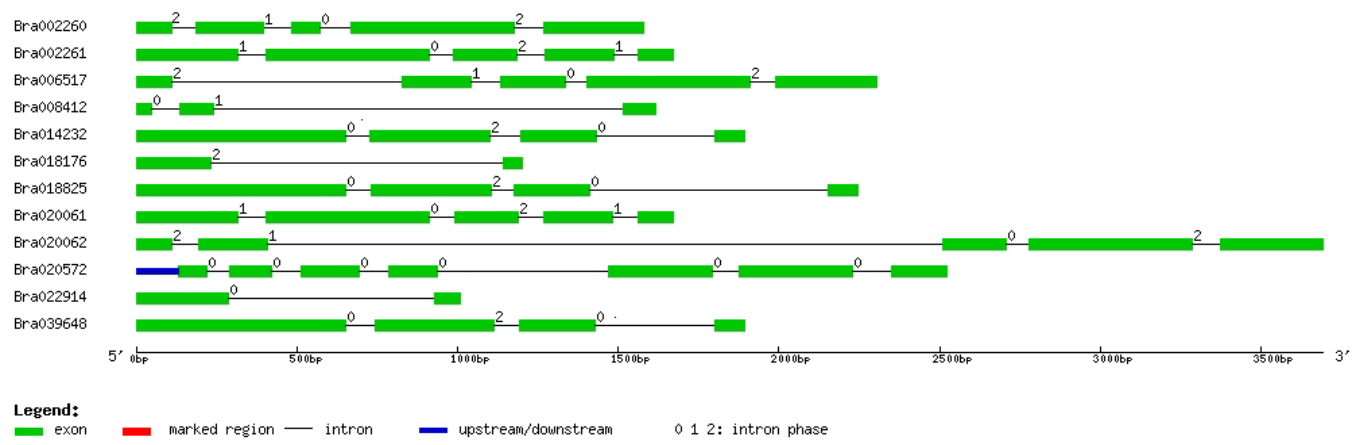

Figure 2. Intron and exon organization of the corresponding BrTUA genes.

\section{Phylogenetic analysis and prediction of molecular function of BrTUA}

The multiple alignments of full-length protein sequences and CDS of BrTUA were used to construct an unrooted neighbor joining phylogenetic tree with the Mega 4.1 software. The 12 BrTUA genes and the TUAs from other plants were classified into five different groups based on their CDS. Bra018825, Bra020572, Bra014232, and Bra039648 belonged to group 1 with the 
genetic relationship of Bra018825, Bra014232, and Bra039648 being closer to AtTUA2, AtTUA4, and AtTU6. Bra002260, Bra002261, Bra006517, Bra020061, and Bra020062 belonged to group 2, with their genetic relationship being closer to AtTUA3 and AtTU5. Bra008412, Bra018176, and Bra022914 were clustered in one group. Only Bra020572 had a close genetic relationship with Zea mays, Eleusine indica, Hordeum vulgare, and Alopecurus aequalis (Figure 3A). The TUA phylogenetic tree reflected the genetic relationship of species, which demonstrated that the evolution of TUA was synchronous with evolution of the species.

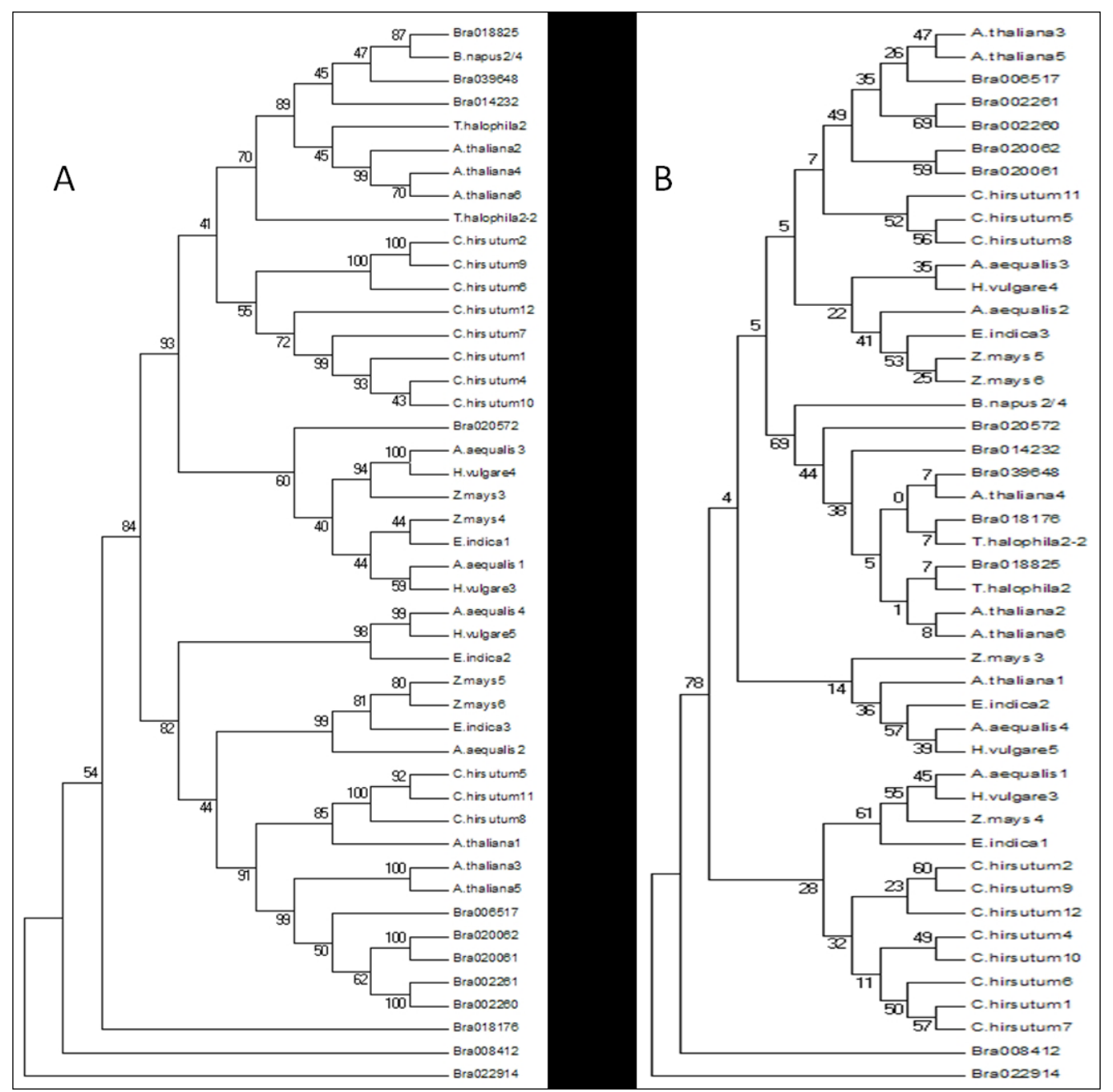

Figure 3. Analysis of genetic evolution of BrTUAs. Neighbor-joining phylogenetic tree based on CDS of the genes (A). Neighbor-joining phylogenetic tree based on the amino acid sequence (B).

The 12 BrTUA genes and the TUAs from other plants were classified into six different groups based on their amino acid sequences. As observed in the case of the phylogenetic tree generated on the basis of CDS, Bra002260, Bra002261, Bra006517, Bra020061, and Bra020062 belonged to 
group 1, which had the closest genetic relationship with AtTUA3 and AtTU5. Bra018825, Bra014232, Bra018176, and Bra039648 belonged to group 2, which had closer genetic relationship with AtTUA2, AtTUA4, and AtTUA6. Among these, Bra018825, Bra039648, and Bra018176 had the closest genetic relationship with AtTUA, AtTUA4, and ThTUA2-2 (Thellungiella halophila a-tubulin2-2), respectively. Based on this phylogenetic tree, the genetic relationships of BrTUAs were relatively distant from the TUAs of Z. mays, Gossypium hirsutum, E. indica, H. vulgare, and A. aequalis (Figure 3B).

The molecular function of the BrTUA proteins was analyzed using the tools available at EMBL-EBI. Among the 12 BrTUA, Bra008412, Bra018176, and Bra022914 lacked a molecular function and the structural domain typical of TUA. The remaining nine BrTUAs had a molecular function and core structural domains of TUA, such as the GTPase and 2-layer sandwich domain and the C-terminal. Among the proteins of those BrTUA genes (copies) that had a molecular function, five (Bra002260, Bra002261, Bra006517, Bra020061, and Bra020062) had an autoregulation site for the binding of $\beta$-tubulin (Table 2). However, Bra002260 did not have a conserved site (an invariant region rich in glycine residues).

\section{Cis-elements in the promoter sequences of BrTUA}

To further understand the transcriptional regulation and potential functions of BrTUA genes, the cis-elements of their promoter sequences were predicted. More than 50 cis-elements that could be classified into 27 types were found in the 1.5-kb upstream region of the BrTUA genes by Plant CARE. Five BrTUAs, which had the molecular function and the auto-regulation binding site for $\beta$-tubulin showed greater differentiation; their promoters had cis-regulatory elements that were involved in circadian regulation and GBF3 factor binding site (Table 3). The five BrTUA promoter sequences contained 12 to 14 cis-elements responsive to light. The low-temperature response cis-element was found in three BrTUA genes. Interestingly, cis-elements involved in hormonal response of plants were significantly different in the five BrTUAs; for example, the abscisic acid response element (ABRE), gibberellin-responsive element (GRE), and MJ-responsive element (TGACG-motif) were found in the different BrTUA promoters, but a cis-acting element involved in gibberellin-responsiveness (TATCbox) was not found in these five BrTUAs. Moreover, four BrTUA genes contained one or more ciselements involved in tissue-specific expression, such as as-2-box, OCT, HD-Zip1, and HD-Zip2. These cis-elements in the BrTUA promoter might be essential in mediating the response of plants to biotic and abiotic stresses as well as during growth and development.

\section{Analysis of gene expression by RT-PCR}

The expression of BrTUA genes, whose proteins were predicted to have molecular function and auto-regulation binding site for $\beta$-tubulin, in various tissues and during different growth stages was analyzed by RT-PCR using gene specific primers (Figure 4A). The relative expression levels were determined after normalization for the GADPH gene expression. The expression of the five BrTUA genes was detected in the floral axis, leaf, flower, and pod whereas weak expression of Bra002260 and Bra006517 was observed in the root. The expression of the five BrTUA genes was not only tissue-specific but the level of expression was also different in the different tissues. The expression level of Bra006517 in leaf, flower, floral axis, and pod was much higher than that of the other four BrTUAs. Moreover, we found that the expression levels of Bra006517, Bra002261, and Bra020062 were relatively higher in leaf than in the other parts. The expression of Bra002260, Bra006517, and Bra002261 in the floral axis exceeded that of Bra020061 and Bra020062. 
Y.W. Zhang et al.

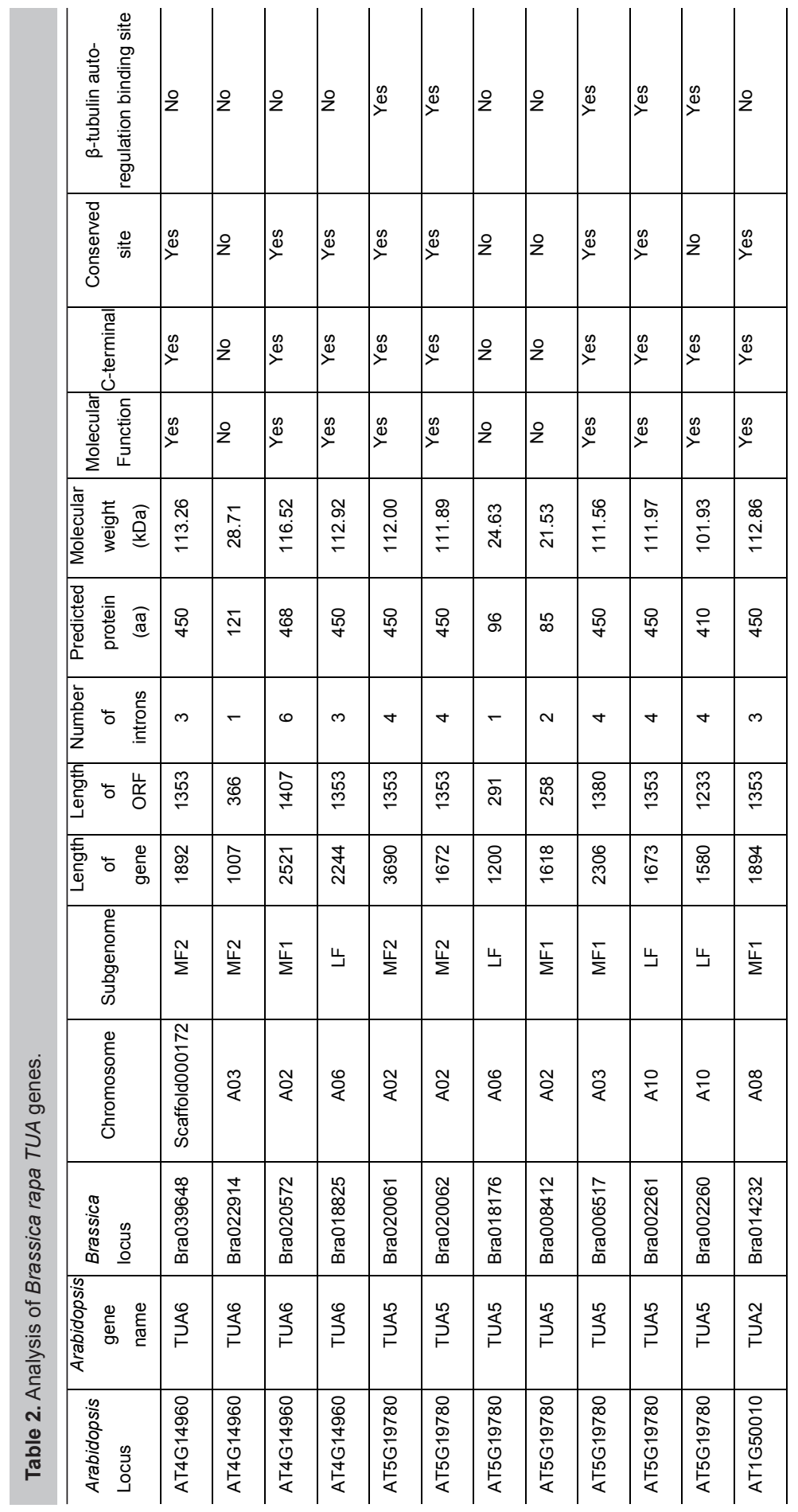


Table 3. Putative partial cis-elements in the promoters of 5 BrTUAs having molecular function and auto-regulation binding site for $\beta$-tubulin.

\begin{tabular}{l|c|c|c|c|c}
\hline cis-regulatory elements & Bra020061 & Bra020062 & Bra006517 & Bra002261 & Bra002260 \\
\hline ABRE & No & Yes & No & Yes & No \\
\hline ARE & Yes & No & Yes & Yes & Yes \\
\hline as-2-box & No & No & No & Yes & No \\
\hline ATGCAAAT motif & No & No & No & Yes & Yes \\
\hline CAT-box & No & Yes & Yes & No & No \\
\hline CGTCA-motif & No & Yes & No & No \\
\hline ERE & No & No & No & Yes & Yes \\
\hline GA-motif & No & No & No & Yes & Yes \\
\hline GARE-motif & No & No & No & Yes & Yes \\
\hline GCN4-motif & Yes & Yes & No & No & No \\
\hline HD-Zip1 & No & No & No & Yes & No \\
\hline HD-Zip2 & No & No & Yes & Yes & No \\
\hline LTR & No & Yes & Yes & No & Yes \\
\hline OCT & No & No & No & Yes & No \\
\hline RY-element & No & No & No & No & Yes \\
\hline Skn-1_motif & Yes & No & Yes & Yes & Yes \\
\hline TCA-element & Yes & No & Yes & No & Yes \\
\hline TGACG-motif & No & Yes & No & Yes & No \\
\hline
\end{tabular}
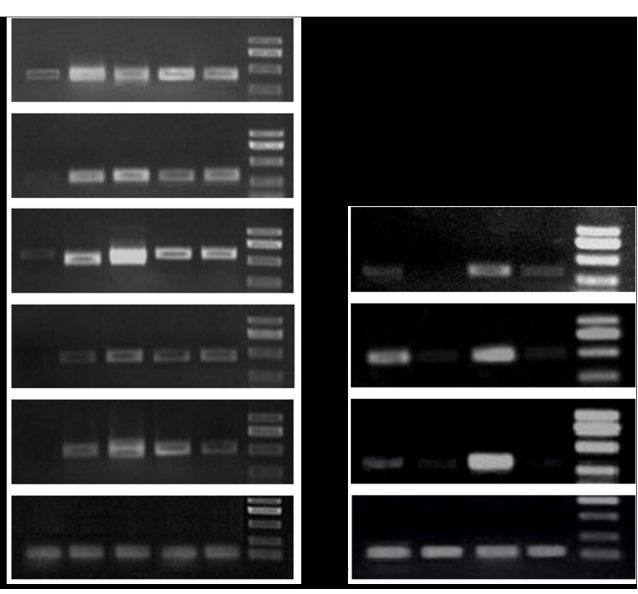

Figure 4. Gene expression of BrTUAs analyzed by RT-PCR. BrTUA expression in different organs (A). a. to f., GADPH, Bra020062, Bra020061, Bra006517, Bra002261, Bra002260, respectively. From left to right in each figure the lanes are for root, floral axis, leaf, flower, and pod, respectively. BrTUA expression during the bolting process (B). a. to d. GADPH, Bra002261, Bra006517, Bra002260, respectively. From left to right in each figure the lanes are for the samples before vernalization, after vernalization, the period of leaf color change, and the period of floral axis elongation. 
In a previous study, we demonstrated that the leaf color changed before bolting and after budding in the Chinese cabbage. After the leaf color changed, flower bud and floral axis of Chinese cabbage began to elongate (Hanet al., 2011). Our results, based on the proteomic analysis done before and after the color change, have revealed that tubulin levels were different during these stages (Zhang YW, Guo MH, Tang XB, Jin D, et al., unpublished data). Based on these observations, Bra002260, Bra002261, and Bra006517, whose expression was higher in the floral axis, were chosen for this experiment. The variations in their expressions were analyzed during the bolting process. The results indicated that the expression patterns of three TUAs were consistent, their expression reduced after vernalization, were high during the period when the leaves changed their color, and they finally reduced during the elongation of the floral axis. During the color-change of leaves, the increase in the expression of Bra002261 and Bra006517 was apparently higher than that of Bra002260, which demonstrated that Bra002261 and Bra006517 played major roles in the process of bolting (Figure 4B).

\section{Influence of allogenic material on BrTUA expression}

To analyze the participation of BrTUA in the regulation of bolting in Chinese cabbage, the effects of $\mathrm{GA}_{3}, \mathrm{MJ}$, and taxol on the floral axis elongation and the expression of Bra002261 and Bra006517 were studied. The floral axis elongation of Chinese cabbage apparently changed after spraying three allogenic materials approximately at the time of leaf color change. The $\mathrm{GA}_{3}$ spray inhibited the floral axis elongation, MJ promoted its elongation, and taxol, which inhibits tubulin, had a lesser effect on the elongation; $\mathrm{MJ}$ in combination with taxol, however, significantly promoted the floral axis elongation (Figure 5A).

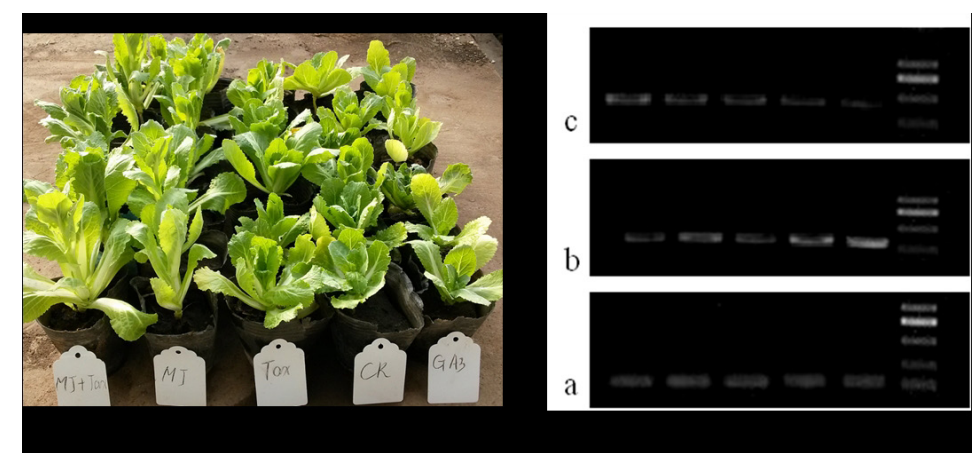

Figure 5. Effect of spraying allogenic materials on bolting and expression of BrTUAs in Chinese cabbage. A. Effect on bolting. From left to right, plants sprayed with methyl jasmonate $(\mathrm{MJ})+\operatorname{taxol}, \mathrm{MJ}$, taxol, control (CK), and $\mathrm{GA}_{3}$, respectively. B. Effect on the expression of BrTUAs. a.-c., GADPH, Bra006517, and Bra002261. From left to right in each picture, the lanes are for samples sprayed with $\mathrm{GA}_{3}, \mathrm{CK}$, taxol, MJ, MJ + Taxol.

Meanwhile, we observed that after spraying $\mathrm{GA}_{3}, \mathrm{MJ}$, and taxol, the expression levels of Bra002261 and Bra006517 changed, and the response of the two TUAs to the three allogenic materials was not completely identical (Figure 5B). After spraying $\mathrm{GA}_{3}$, the expression of Bra006517 was weakly down-regulated, whereas that of Bra002261 was weakly up-regulated. After spraying MJ, the expression patterns of the two TUAs were contrary to those observed for the $\mathrm{GA}_{3}$ spray, and after the taxol spray, the expression of both the TUAs was weakly down-regulated. The spraying of $\mathrm{MJ}$ and taxol at the same time resulted in significantly up-regulation of Bra006517 expression 
whereas the expression of Bra002261 was weakly down-regulated. The different response of the two TUAs to $\mathrm{GA}_{3}$, MJ, and taxol might have been related to an upstream cis-acting element, which still needs to be extensively explored.

\section{DISCUSSION}

\section{Characterization and evolution of the BrTUA genes in B. rapa}

In the present study, we identified 12 members of the TUA gene family in Chinese cabbage. Compared to the $A$. thaliana TUA gene family that contains 6 genes, the TUA gene family of Chinese cabbage did not appear to have undergone obvious expansion after the evolution, including a process of triploidization and double-ploidization during the evolutionary process as a new polyploidy (Cheng et al., 2013). In terms of the structure of the BrTUA gene family, some BrTUAs lost big fragments during the process of diploidization, leading to the loss of molecular function and typical structural domains in Bra018176, Bra008412, and Bra022914, and that of auto-regulation binding site for $\beta$-tubulin in Bra039648, Bra020572, Bra014232, and Bra018825. Zhang et al. (2009) reported that after gene duplication, the exon splicing element rapidly varied in different copies and the differentiation of noncoding region was apparently greater than that of the coding region. In the present study, we also found that the promoters and introns of the BrTUA gene family changed a lot during the long process of evolution. Although most BrTUA retained four introns as in the case of AtTUA, the length ranged from 65 to $2100 \mathrm{bp}$ and a 128-bp regulatory sequence was observed to be inserted ahead of the first codon in Bra020572. The position of introns also changed significantly; they were distributed in every region of the genes, whereas introns of plant microtubule genes were mostly distributed in the 5 '-end (Dibb and Newman, 1989; Sakurai et al., 2002).

\section{Diverse cis-elements in the promoters of BrTUA genes}

The upstream cis-elements of BrTUAs revealed huge differences among the different copies, which differed in number and kinds. The differentiation of cis-acting element in the upstream regulatory regions could have followed the evolution of $B$. rapa genome. Kassahn et al. (2009) and Conant (2010) reported that the cis-acting element in the upstream regulatory regions would have rapid variation after the genome evolution and gene duplication. The evolution of $B$. rapa genome inevitably led to recombination and loss of BrTUAs cis-acting element.

Hormones are the basis of plant growth and development. Growth conditions of cells can be changed by regulating the organizational behavior of cortical microtubule; for example, $\mathrm{GA}_{3}$ and $A B A$ could regulate the cell elongation through changing the array direction of the microtubules (Mendu and Silfow, 1993; Sakiyama-Sogo and Shibaoka, 1993). In the present study, we observed that the upstream regulatory region of BrTUAs had numerous cis-elements involved in plant hormone response, such as ABRE, GRE, and MJ responsive element (TGACG-motif). Therefore, we sprayed $\mathrm{GA}_{3}$ and $\mathrm{MJ}$, approximately near the period of leaf color change, to investigate their effects on the expression of BrTUAs and on the floral axis elongation. The results showed that $\mathrm{GA}_{3}$ restrained the floral axis elongation. In a previous study, during the period of leaf color change in Chinese cabbage, $\mathrm{GA}_{3}$ content reached its peak (Hanet al., 2011). Thus, spraying $\mathrm{GA}_{3}$, near the period of leaf color change, could lead to very high $\mathrm{GA}_{3}$ content that could restrain bolting. It is reported that after MJ spray on A. thaliana, the expression levels of AtGA3 and AtGA20, which are the key genes of compounding, the gibberellin activity apparently reduced, resulting in late 
flowering (Lin et al., 2011). However, in our experiments we found that spraying MJ could speed up the floral axis elongation and lead to early flowering of Chinese cabbage. This might be related to MJ-responsive element (TGACG-motif) in the promoter of Bra002261. Spraying MJ induced the expression of BrTUA (Bra002261), changed the arrangement of microtubules, promoted cell elongation, and accelerated the floral axis elongation.

\section{Involvementof BrTUAs in regulation of bolting}

Microtubule is a ubiquitous structure in the cytoskeleton, containing a-tubulin as the basic unit. Different isomers of $\alpha$-tubulin protein genes have different expression patterns during a specific developmental stage or in the cells of specific tissues (Carpenter et al., 1992; Xu et al., 1999; Li et al., 2007). We had previously observed the specific expression of $\alpha$-tubulin in the process of leaf color change in Chinese cabbage after the floral bud differentiation, using proteomic analysis (Zhang YW, Guo MH, Tang XB, Jin D, et al., unpublished results). Based on this observation, we could associate the variation of microtubule arrangement with the floral axis elongation and bolting after the floral bud differentiation. We analyzed the Bra002260, Bra002261, and Bra006517 expression during the process of vernalization and bolting in Chinese cabbage. The results revealed that the expression of the three TUAs changed with developmental process. The expression level was greatest during the period of leaf color change before bolting, which demonstrated the process of floral axis elongation and bolting needs a-tubulin protein. The TUAs were up-regulated after vernalization. This result was consistent with the findings of Mei et al. (2011), where they observed a differential expression of microtubular protein specificity $C$ partner gene during winter, which is related to unfolding of the microtubular protein, and has the function of regulating the microtubular protein structure. It was found that after spraying $\mathrm{GA}_{3}$ and $\mathrm{MJ}$, the speed of floral axis elongation in the Chinese cabbage changed along with the expression of TUAs. The results presented in this report, establish the involvement of BrTUA genes in the regulation of bolting in Chinese cabbage. However, spraying taxol had little effect on BrTUA expression and on the speed of floral axis elongation during the period of color change in leaves, which might be due to the change in the arrangement of microtubules during this period.

\section{Conflicts of interest}

The authors declare no conflict of interest.

\section{ACKNOWLEDGMENTS}

Research supported by the Foundation for Key Technologies R\&D Program (\#2012BAD02B01-13), the National Natural Science Foundation of China (\#31401895), and the Natural Science Foundation of Heilongjiang Province of China (\#C201221).

\section{REFERENCES}

Carpenter JL, Ploense SE, Snustad DP and Silflow CD (1992). Preferential expression of an $\alpha$-tubulin gene of Arabidopsis in pollen. Plant Cell 4: 557-571.

Chan J, Eder M, Crowell EF, Hampson J, et al. (2011). Microtubules and CESA tracks at the inner epidermal wall align independently of those on the outer wall of light-grown Arabidopsis hypocotyls. J. Cell Sci. 124: 1088-1094. http://dx.doi.org/10.1242/jcs.086702 
Cheng F, Mandáková T, Wu J, Xie Q, et al. (2013). Deciphering the diploid ancestral genome of the Mesohexaploid Brassica rapa. Plant Cell 25: 1541-1554.http://dx.doi.org/10.1105/tpc.113.110486

Conant GC (2010). Rapid reorganization of the transcriptional regulatory network after genome duplication in yeast. Proc. Biol. Sci. 277: 869-876.http://dx.doi.org/10.1098/rspb.2009.1592

Dai ZL, Pan YP, Xiao Y, Qin WB, et al. (2010). Effects of gibberellin treatments at different concentrations on bolting and flowering of common head cabbage. Acta Agric. Shanghai 26: 69-71.

Dibb NJ and Newman AJ (1989). Evidence that introns arose at proto-splice sites. EMBO J. 8: 2015-2021.

Dixon DC, Seagull RW and Triplett BA (1994). Changes in the accumulation of $\alpha$ - and $\beta$-tubulin isotypes during cotton fiber development. Plant Physiol. 105: 1347-1353.

Han DP, Li CG and Zhang YW (2011). Physiological changes before and after leaf color transition in the bolting process of Chinese cabbage. China Veget. 1: 34-38.

Kassahn KS, Dang VT, Wilkins SJ, Perkins AC, et al. (2009). Evolution of gene function and regulatory control after wholegenome duplication: comparative analyses in vertebrates. Genome Res. 19: 1404-1418. http://dx.doi.org/10.1101/gr.086827.108

Kopczak SD, Haas NA, Hussey PJ, Silflow CD, et al. (1992). The small genome of Arabidopsis contains at least six expressed a-tubulin genes. Plant Cell 4: 539-547.

Li L, Wang XL, Huang GQ and Li XB (2007). Molecular characterization of cotton GhTUA9 gene specifically expressed in fibre and involved in cell elongation. J. Exp. Bot. 58: 3227-3238. http://dx.doi.org/10.1093/jxb/erm167

Lin CC, Chu CF, Liu PH, Lin HH, et al. (2011). Expression of an Oncidium gene encoding a patatin-like protein delays flowering in Arabidopsis by reducing gibberellin synthesis. Plant Cell Physiol. 52: 421-435. http://dx.doi.org/10.1093/pcp/pcq206

Mei JF, Tang CQ, Xu DL, et al. (2011). Study on diggerential gene expression of tea plant (Camellia sinensis) during cold acclimation in winter. Chin. J. Tropical Crops. 32: 648-652.

Mendu N and Silfow CD (1993). Elevated levels of tubulin transcripts accompany the $\mathrm{GA}_{3}$-induced elongation of oat internode segments. Plant Cell Physiol. 34: 973-983.

Oakley RV, Wang YS, Ramakrishna W, Harding SA, et al. (2007). Differential expansion and expression of $\alpha$ - and $\beta$-tubulin gene families in Populus. Plant Physiol. 145: 961-973. http://dx.doi.org/10.1104/pp.107.107086

Parrotta L, Cai G and Cresti M (2010). Changes in the accumulation of $\alpha$ - and $\beta$-tubulin during bud development in Vitis vinifera L. Planta 231: 277-291. http://dx.doi.org/10.1007/s00425-009-1053-9

Sakiyama-Sogo M and Shibaoka H (1993). Gibberellin $A_{3}$ and abscisic acid cause the reorientation of cortical microtubules in epicotyl cells of the decapitated dwarf pea. Plant Cell Physiol. 34: 431-437.

Sakurai A, Fujimori S, Kochiwa H, Kitamura-Abe S, et al. (2002). On biased distribution of introns in various eukaryotes. Gene 300: 89-95. http://dx.doi.org/10.1016/S0378-1119(02)01035-1

Thitamadee S, Tuchihara K and Hashimoto T (2002). Microtubule basis for left-handed helical growth in Arabidopsis. Nature 417: 193-196. http://dx.doi.org/10.1038/417193a

Xu L, Zheng WZ, Zuo ZH, et al. (1999). Progress in molecular biological studies of a-tubulin in maize. Chin. B. Botany 16: 488-494.

Zhang Z, Zhou L, Wang P, Liu Y, et al. (2009). Divergence of exonic splicing elements after gene duplication and the impact on gene structures. Genome Biol. 10: R120. http://dx.doi.org/10.1186/gb-2009-10-11-r120 